\title{
miR-143 inhibits oncogenic traits by degrading NUAK2 in glioblastoma
}

\author{
TING-GANG FU ${ }^{1}$, LING WANG ${ }^{2}$, WEI LI ${ }^{3}$, JIAN-ZHONG LI ${ }^{4}$ and JIAN $\mathrm{LI}^{2}$ \\ ${ }^{1}$ Department of Neurosurgery, Yishui Central Hospital, Linyi, Shandong 276400; ${ }^{2}$ Department of Neurosurgery, \\ Linyi People's Hospital, Linyi, Shandong 276003; ${ }^{3}$ Intensive Care Unit (ICU), Lanling People's Hospital, Lanling, \\ Shandong 277700; ${ }^{4}$ Intensive Care Unit (ICU), Yishui Central Hospital, Linyi, Shandong 276400, P.R. China
}

Received June 3, 2015; Accepted January 7, 2016

DOI: $10.3892 /$ ijmm.2016.2562

\begin{abstract}
Despite evidence that the crucial role of NUAK family, SNF1-like kinase, 2 [NUAK2; also known as sucrose non-fermenting 1 (SNF1)-like kinase (SNARK)], has been highlighted in cancer development and in tumor progression, to the best of our knowledge, there are no studies available to date on the role of NUAK 2 in glioblastoma. Thus, in this study, in order to determine the role of NUAK2 in glioblastoma, we performed western blot analysis to detect its expression in glioma. The results demonstrated that NUAK2 expression was upregulated in glioma tissues and that its expression was associated with the advanced stages of the disease. In vitro, NUAK2 overexpression promoted the proliferation, migration and invasion of A172 glioblastoma cells, whereas the silencing of NUAK 2 with a plasmid carrying shRNA targeting NUAK2 inhibited the proliferation of A172 glioblastoma cells. Moreover, NUAK2 regulated cancer stem cell (CSC)-related gene expression in the glioblastoma cells. We performed an analysis of potential microRNA (miR or miRNA) target sites using 3 commonly used prediction algorithms, miRanda, TargetScan and PicTar. All 3 algorithms predicted that miR-143 targeted the 3'-untranslated region (3'UTR) of NUAK2. Subsequent experiments confirmed this prediction. Finally, we found that miR-143 inhibited the proliferation, migration and invasion of the glioblastoma cells. Thus, the findings of the present study demonstrate that miR-143 inhibits oncogenic traits by degrading NUAK2 in glioblastoma.
\end{abstract}

\section{Introduction}

Glioblastoma is the most common primary malignant brain tumor in adults with overall survival rates of $<3.3 \%$ at

Correspondence to: Dr Jian Li, Department of Neurosurgery, Linyi People's Hospital, 27 Jiefang Road, Linyi, Shandong 276003, P.R. China

E-mail: jianli90@yahoo.com

Key words: miR-143, NUAK family, SNF1-like kinase, 2, glioblastoma, cancer stem cells
5 years (1). Few effective treatments are available. Following maximal surgical tumor resection, the current standard of care is based on a phase 3 , randomized clinical trial conducted by the European Organization for Research and Treatment of Cancer and the National Cancer Institute of Canada, which demonstrated that concurrent treatment with daily doses of temozolomide (TMZ) and radiotherapy followed by maintenance with TMZ was superior to radiotherapy alone (2). Even though this combined chemoradiotherapy approach led to improved outcomes, few patients survive beyond 5 years (3). Thus, determining the molecular mechanisms responsible for the development and progression of the disease, as well as the development of radiation resistance, may broaden our understanding of the pathogenesis and progression of the disease and may provide novel therapeutic strategies.

The sucrose non-fermenting 1 (SNF1)/AMP-activated protein kinase (AMPK) family functions to control the balance of cellular metabolism, and is activated by the cellular AMP:ATP ratio that is regulated by metabolic stresses, such as hypoxia and glucose deprivation $(4,5)$. Twelve protein kinases [brain-specific serine/threonine-protein kinase (BRSK)1, BRSK2, NUAK family, SNF1-like kinase, 1 (NUAK1), NUAK family, SNF1-like kinase, 2 (NUAK2) which is also known as SNF1-like kinase (SNARK), salt-inducible kinase 2 (QIK), serine/threonine-protein kinase SIK3 (QSK), salt-inducible kinase (SIK), MAP/microtubule affinity-regulating kinase (MARK)1, MARK2, MARK3, MARK4 and maternal embryonic leucine zipper kinase (MELK)] have been identified as AMPK- $\alpha 1$ - and AMPK- $\alpha 2$-related kinases in the human kinome $(6,7)$. AMPK-related kinases function as critical sensors coupling cellular energy status to cell growth and proliferation by modulating the cell-cycle machinery and, when deregulated, they result in cancer development and tumor progression in several types of cancer of different cell lineages $(4,8,9)$. NUAK2, which resides at 1q32, is a member of the SNF1/AMPK family (serine/threonine kinases) that is regulated by the putative tumor suppressor, liver kinase B1 (LKB1; also known as serine/threonine kinase 11), as well as by death receptor signaling through nuclear factor $(\mathrm{NF})-\kappa \mathrm{B}(6,10-12)$. The crucial role of NUAK2 has been highlighted in cancer development and in tumor progression $(6,11,12)$. Although AMPK has anti-oncogenic properties, its role in glioma has not yet been reported to date, to the best of our knowledge. 
MicroRNAs (miRs or miRNAs) are small, non-coding RNAs that modulate protein expression by binding to complementary or partially complementary sequences in the $3^{\prime}$ untranslated region (3'UTR) of target mRNAs and thereby target mRNAs for degradation or translational inhibition $(13,14)$. miR-143 is downregulated in glioma tissues and directly targets the neuroblastoma RAS viral oncogene homolog (N-RAS) and functions as a tumor suppressor in the disease (15). The overexpression of miR-143 has been shwon to decrease the expression of N-RAS, inhibit phosphoinositide 3-kinase $(\mathrm{PI} 3 \mathrm{~K}) / \mathrm{AKT}$ and mitogen-activated protein kinase (MAPK)/extracellular signal-regulated kinase (ERK) signaling, and to attenuate the accumulation of p65 in the nuclei of glioma cells, as well as to decrease migration, invasion, tube formation and attenuate tumor growth and angiogenesis (15). miR-143 has also been shown to sensitize glioma cells to TMZ, the first-line drug for glioma treatment (15). However, the mechanisms through which miR-143 functions as a tumor suppressor gene have not yet been fully elucidated.

In this study, we demonstrate that NUAK2 expression is upregulated in glioma tissues and that its expression is associated with the advanced stages of the disease. In vitro, NUAK2 overexpression promoted the proliferation, migration and invasion of A172 glioblastoma cells, whereas the silencing of NUAK2 with a plasmid carrying shRNA targeting NUAK2 inhibited glioma cell proliferation. Moreover, NUAK2 regulated cancer stem cell (CSC)-related gene expression in glioma cells. We also performed an analysis of potential miRNA target sites using 3 commonly used prediction algorithms, miRanda, TargetScan and PicTar. All 3 algorithms predicted that miR-143 targets the 3'UTR of NUAK2. Subsequent experiments confirmed this prediction. Finally, we found that miR-143 inhibited the proliferation, migration and invasion of glioblastoma cells. Thus, the findings of our study demonstrate that miR-143 inhibits oncogenic traits by degrading NUAK2 in glioblastoma.

\section{Materials and methods}

Glioma tissues, cells and NUAK2 expression plasmids. Sixteen tissue samples of human glioma tissue (4 samples of WHO grade I and 12 samples of WHO grade IV) and matched adjacent normal tissue samples were obtained from the Department of Neurosurgery, Yishui Central Hospital (Linyi, China). The mean patient age was 56 years (range, $31-78$ years). The use of human tissue samples followed internationally recognised guidelines, as well as local and national regulations. The medical ethics committee approved the experiments undertaken. Informed consent was obtained from each individual. The human glioblastoma cell line, A172, was kindly donated by Dr Yong Yu (Hubei Cancer Center, Wuhan, China). The A172 glioblastoma cells were maintained in DMEM supplemented with $10 \%$ fetal bovine serum (FBS). The NUAK2 expression plasmid, the plasmid carrying shRNA targeting NUAK2 (shNUAK2), the empty vector and the scrambled shRNA were purchased from Tiangen Biotech (Beijing, China).

miRNA precursors. The miR-143 miRNA precursor (pre-miR-143) and a control precursor (control miR) were purchased from Ambion, Inc. (Austin, TX, USA).
Cell transfection. For the transfection experiments, the cells were cultured in serum-free medium without antibiotics at $60 \%$ confluence for $24 \mathrm{~h}$, and then transfected with the NUAK2 expression plasmid, shNUAK2, pre-miR-143 or control miR using transfection reagent (Lipofectamine 2000; Invitrogen, Carlsbad, CA, USA) according to the manufacturer's instructions. Following incubation for $6 \mathrm{~h}$, the medium was removed and replaced with normal culture medium for $48 \mathrm{~h}$, unless otherwise specified.

Western blot analysis. Western blot analysis was performed as described in a previous study (16). Briefly, following incubation with anti-NUAK2 (1:500; ab107287), anti-enhancer of zeste 2 polycomb repressive complex 2 subunit (EZH2; 1:500; ab186006), anti-L1 cell adhesion molecule (L1CAM; 1:500; ab208155), anti-CD133 (1:500; ab16518), anti-Bmi (1:500; ab38295), anti-multidrug resistance protein 1 (MDR1; 1:500; ab170904), anti-stage-specific embryonic antigen 1 (SSEA1; 1:500; sc-21702), anti-signal transducer and activator of transcription 3 (STAT3; 1:500; ab68153) and anti- $\beta$-actin (1:500; ab179467/ab3280) primary antibodies (all purchased from Abcam, Cambridge, MA, USA) overnight at $4^{\circ} \mathrm{C}$, IRDye $^{\mathrm{TM}}-800$ conjugated anti-rabbit/anti-mouse secondary antibodies (LI 926-32211/LI 926-32210; Li-COR Biosciences, Lincoln, NE, USA) were used for $30 \mathrm{~min}$ at room temperature. The specific proteins were visualized using the Odyssey ${ }^{\mathrm{TM}}$ Infrared Imaging System (Li-COR Biosciences).

3-(4,5-Dimethylthiazol-2-yl)-2,5-diphenyltetrazolium (MTT) cell proliferation assay. Cell proliferation was assessed by MTT assay (Sigma, St. Louis, MO, USA). The cells were seeded into a 96 well-plate at 4,000 cells per well and measured using an MTT kit according to the manufacturer's instructions (Sigma). The absorbance was directly proportional to the number of viable cells.

BrdU cell proliferation assay. Cell proliferation was also assessed using a colorimetric BrdU proliferation kit according to the manufacturer's instructions (Roche, Indianapolis, IN, USA). The transfected cells were labeled with BrdU for $4 \mathrm{~h}$. Genomic DNA was fixed and denatured, followed by incubation with peroxidase-conjugated anti-BrdU antibody for $90 \mathrm{~min}$. A substrate for the conjugated peroxidase was then added and the reaction product was quantified by measuring the absorbance. The results were then normalized to the number of total viable cells.

Cell migration and invasion assay. For Transwell migration assays, $2.5 \times 10^{4}$ cells were plated in the top chamber with the non-coated membrane (24-well insert; pore size, $8 \mathrm{~mm}$; BD Biosciences, San Jose, CA, USA). For cell invasion assays, $1.25 \times 10^{5}$ cells were plated in the top chamber with Matrigel-coated membrane (24-well insert; pore size, $8 \mathrm{~mm}$; BD Biosciences). In both assays, the cells were plated in medium without serum or growth factors, and the medium supplemented with serum was used as a chemoattractant in the lower chamber. The cells were incubated for $24 \mathrm{~h}$ and the cells that did not migrate or invade through the pores were removed using a cotton swab. The cells on the lower surface of the membrane were stained with the Diff-Quick stain set 

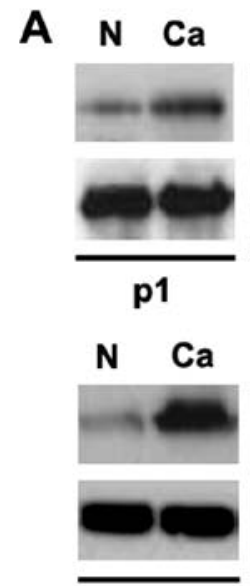

p7

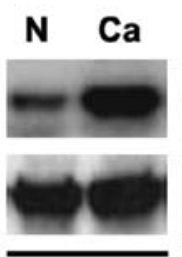

p2

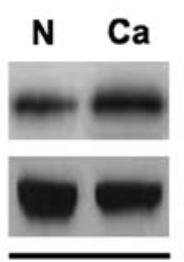

p3

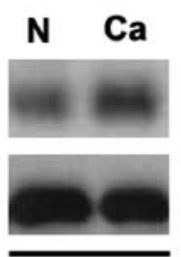

p4

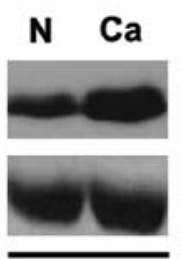

p5

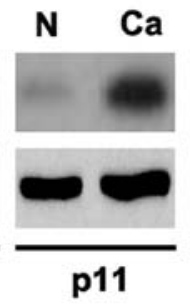

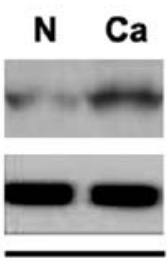

p6

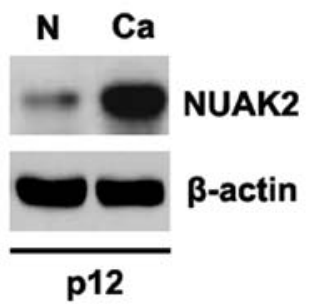

B

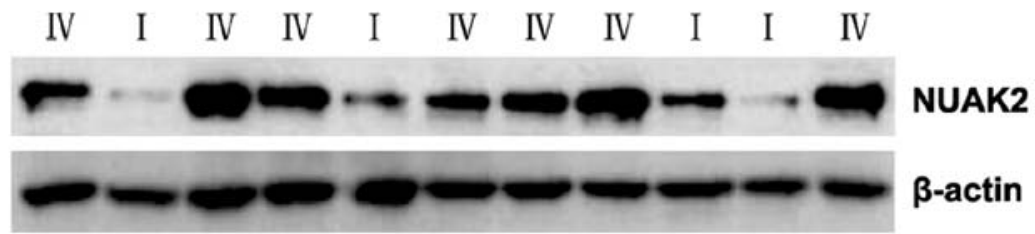

Figure 1. Aberrant NUAK family, SNF1-like kinase, 2 (NUAK2) expression in glioblastoma. (A) Western blot analysis of NUAK2 protein expression in glioma tissues $(\mathrm{Ca})$ and adjacent normal tissues $(\mathrm{N})$. $\beta$-actin was used as a loading control; $\mathrm{n}=12$ samples (WHO grade IV). (B) Western blot analysis of NUAK2 protein expression in advanced glioblastoma tissues (IV) and low grade glioma (I). $\beta$-actin was used as a loading control; $\mathrm{n}=11$ samples (7 samples from 12 in 'A' + 4 WHO grade I samples).

(Dade Behring, Newark, NJ, USA) and counted under a Zeiss microscope (Zeiss, New York, NY, USA).

Bioinformatics analysis. The analysis of potential miRNA target sites was performed using the following 3 commonly used prediction algorithms: TargetScan (http://www.targetscan. org), miRanda (http://www.microrna.org/microrna/home.do) and PicTar (http://pictar.mdc-berlin.de/).

Immunofluorescence staining. To perform immunofluorescence staining, the A172 cells were plated on glass coverslips in 6-well plates and transfected with $50 \mathrm{nM}$ pre-miR-143 or control miR. At $36 \mathrm{~h}$ following transfection, the coverslips were stained with the above-mentioned anti-NUAK2 antibody. Alexa Fluor 488 goat anti-rabbit IgG antibody (ab150077; Abcam) was used as a secondary antibody. The coverslips were counterstained with DAPI (Invitrogen-Molecular Probes, Eugene, OR, USA) for visualization of the nuclei. Microscopic analysis was performed with a confocal laser-scanning microscope (Leica Microsystems, Bensheim, Germany). The fluorescence intensities were measured in a few viewing areas for 200-300 cells/coverslip and analyzed using ImageJ $1.37 \mathrm{v}$ software (http://rsb.info.nih.gov/ij/index.html).

Reverse transcription-quantitative polymerase chain reaction (RT-qPCR) for NUAK2. Total RNA was isolated from the cells using TRIzol reagent (Invitrogen). First-strand cDNA was synthesized from the total RNA using M-MLV reverse transcriptase (Promega, Madison, WI, USA) and random hexamer primers (Sangon, Shanghai, China). The thermal cycling conditions were as follows: denaturation for $30 \mathrm{sec}$ at $95^{\circ} \mathrm{C}$, annealing for $45 \mathrm{sec}$ at $52-58^{\circ} \mathrm{C}$ depending on the primers used, and extension for $45 \mathrm{sec}$ at $72^{\circ} \mathrm{C}$. The PCR products were visualized on $2 \%$ agarose gels stained with ethidium bromide under UV transillumination. qPCR was performed with a Power SYBR-Green PCR Master Mix (Applied Biosystems, Carlsbad, CA, USA) according to the manufacturer's instructions. The primer sequences were as follows: NUAK2 forward, 5'-CTGAGACTGATAACGAGGAT-3' and reverse, 5'-GAGGTGTTTCTGCTTGAC-3'.

$R T$-qPCR for miRNAs. Total RNA from the cultured cells, with the efficient recovery of small RNA, was isolated using the mirVana miRNA isolation kit (Ambion, Inc.). The detection of the mature form of miRNAs was performed using the mirVana qRT-PCR miRNA detection kit, according to the manufacturer's instructions (Ambion, Inc.). U6 small nuclear RNA was used as an internal control.

Statistical analysis. Data are expressed as the means $\pm \mathrm{SE}$, with the number of independent experiments $(n=3)$, and were analyzed using the Student's t-test. A $p<0.05$ was considered to indicate a statistically significant difference.

\section{Results}

Aberrant NUAK2 expression in glioma tissues. To assess the expression of NUAK2 in glioma tissues, western blot analysis was conducted on 12 pairs of glioblastoma tissues and matched adjacent normal tissue samples. The expression of NUAK2 was consistently higher in the glioblastoma tissues than in the normal tissues (Fig. 1A). Moreover, the analysis 
A

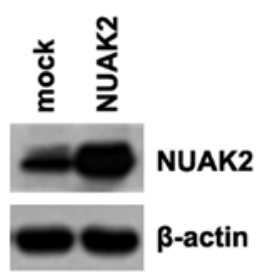

B

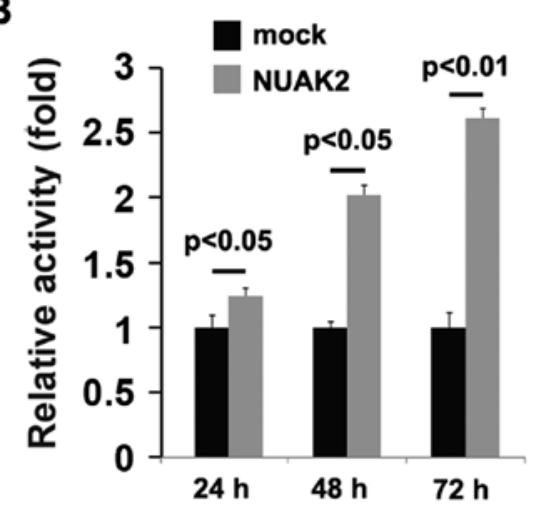

C

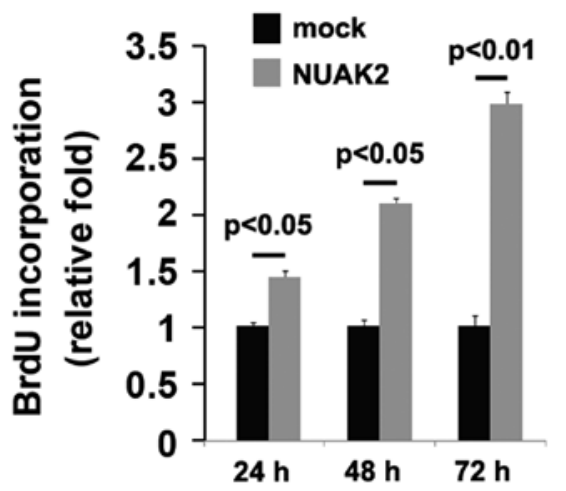

D

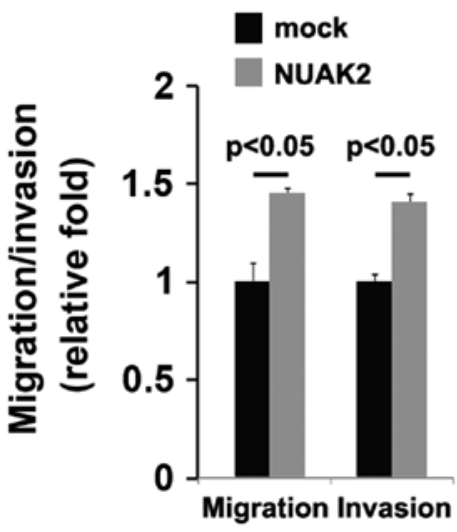

Figure 2. NUAK family, SNF1-like kinase, 2 (NUAK2) overexpression promotes the proliferation, migration and invasion of A172 glioblastoma cells. (A) Western blot analysis of NUAK 2 expression in A172 cells. A172 cells were transfected with a NUAK2 expression plasmids or the empty vector (mock). $\beta$-actin was used as a loading control. $n=3$ experiments. (B) MTT assay of A172 cells. A172 cells were transfected with a NUAK2 expression plasmid or the empty vector (mock) and then cell viability was measured at the indicated time points by MTT assay. $n=3$ experiments. (C) Brdu incorporation assay of A172 cells. A172 cells were transfected with the NUAK2 expression plasmid or the empty vector (mock). $n=3$ experiments. (D) Matrigel invasion assay and Transwell migration assay of A172 cells transfected with the NUAK2 expression plasmids or empty vector (mock). $n=3$ experiments. of NUAK2 expression in the tissues from patinets with highgrade (WHO grade IV) gliomas (namely glioblastoma) and low-grade (WHO grade I) gliomas revealed that NUAK2 was upregulated in the advanced stages of the disease (Fig. 1B). These data support the notion that NUAK2 functions as an oncogene in glioma tissues.

The overexpression of NUAK2 promotes the proliferation, migration and invasion of A172 glioblastoma cells. In an attempt to determine the role of NUAK2 in regulating the proliferation of A172 cells, the cells were transfected with a NUAK2 expression plasmid. We found that the NUAK2 protein levels were significantly increased in the cells transfectd with the NUAK2 expression plasmid compared to the cells transfected with the emtpy vector (mock; Fig. 2A). Following stable transfection, the proliferation rates of the A172 cells were determined by MTT assay. The results revealed that the overexpression of NUAK2 significantly increased the proliferation rate of the A172 cells and that the increase in cell proliferation was time-dependent (Fig. 2B). This was further confirmed by Brdu incorporation assay, which indicated that transfection of the cells with NUAK2 resulted in increased DNA synthesis activity per viable cell in the A172 cells in a time-dependent manner (Fig. 2C).

Given that NUAK2 markedly promoted A172 cell proliferation, we then sought to determine whether NUAK2 would have an impact on the migration and invasion of A172 cells. The migration and invasion assay of the A172 cells revealed that NUAK2 overexpression not only promoted the migration, but also promoted the invasion of the A172 cells (Fig. 2D).

The silencing of NUAK2 inhibits the proliferation, migration and invasion of A172 glioblastoma cells. In order to further determine the role of NUAK2 in regulating the proliferation of A172 cells, the cells were transfected with a plasmid carrying shRNA targeting NUAK2 (shNUAK2). We found that the NUAK2 protein levels were significantly decreased following transfection with shNUAK2 (Fig. 3A). Following stable transfection, the proliferation rates of the A172 cells were determined by MTT assay. The results revealed that the silencing of NUAK2 significantly suppressed the proliferation rate of the A172 cells and that the decrease in cell proliferation was time-dependent (Fig. 3B). This was further confirmed by Brdu incorporation assy, which indicated that transfection with shNUAK2 resulted in decreased DNA synthesis activity per viable cell in the A172 cells, in a time-dependent manner (Fig. 3C).

Given that the silencing of NUAK2 markedly inhibited A172 cell proliferation, we then sought to determine whether silencing NUAK 2 would have an impact on the migration and invasion of the A172 cells. The migration and invasion assay of the A172 cells revealed that the silencing of NUAK2 not only suppressed the migration, but also inhibited the invasion of the A172 cells (Fig. 3D).

NUAK2 regulates CSC-related protein expression in A172 glioblastoma cells. We demonstrated the aberrant expression of NUAK2 was found in glioblastoma tissues, and that NUAK2 promoted the proliferation, migration and invasion of A172 cells. It has been reported that there is an association between proliferation, migration and invasion, and CSCs (17-19). Thus, 
A

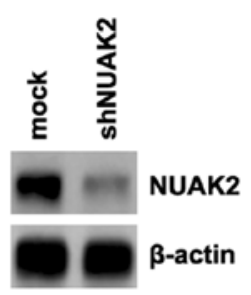

B

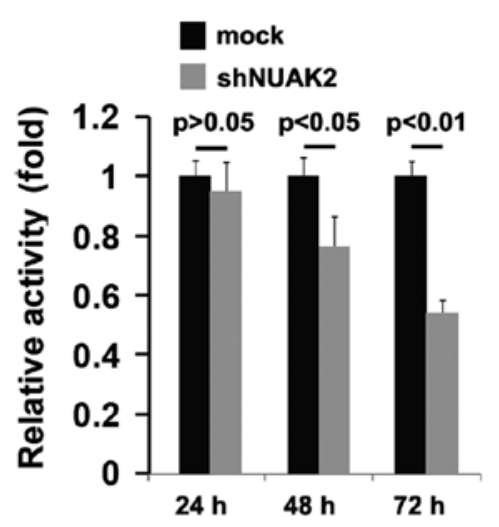

C

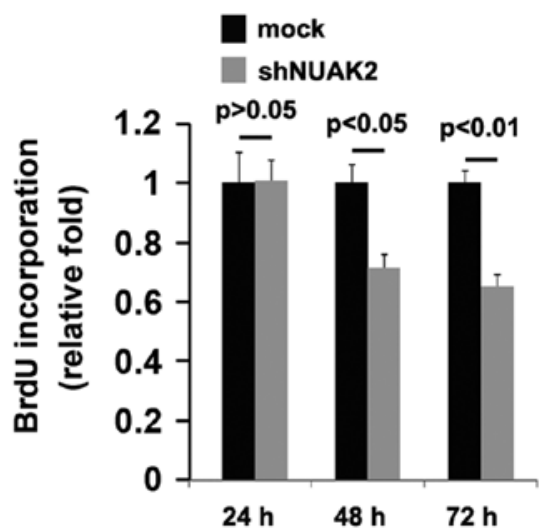

D

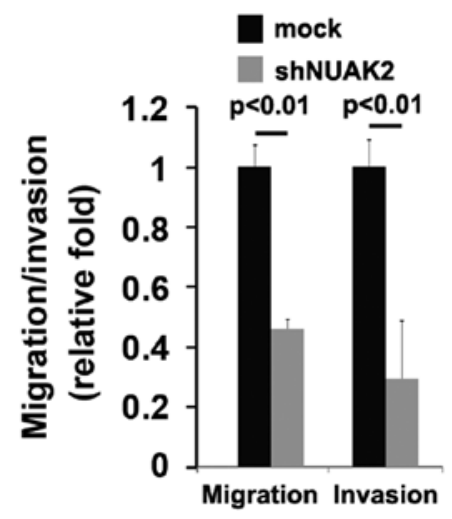

Figure 3. Silencing of NUAK family, SNF1-like kinase, 2 (NUAK2) inhibits the proliferation, migration and invasion of A172 glioblastoma cells. (A) Western blot analysis of NUAK2 in A172 cells. A172 cells were transfected with shNUAK2 plasmids or scramble (mock). $\beta$-actin was a loading control. $\mathrm{n}=3$. (B) MTT assay for A172 cells. A172 cells were transfected with a plasmid carrying shRNA targeting NUAK2 (shNUAK2) or scrambled shRNA (mock) and cell viability was then measured at the indicated time points by MTT assay. $n=3$ experiments. (C) Brdu incorporation assay for A172 cells. A172 cells were transfected with shNUAK2 or the scrambled shRNA (mock). $\mathrm{n}=3$ experiments. (D) Matrigel invasion assay and Transwell migration assay for A172 cells transfected with shNUAK2 or scrambled shRNA (mock). $n=3$ experiments.

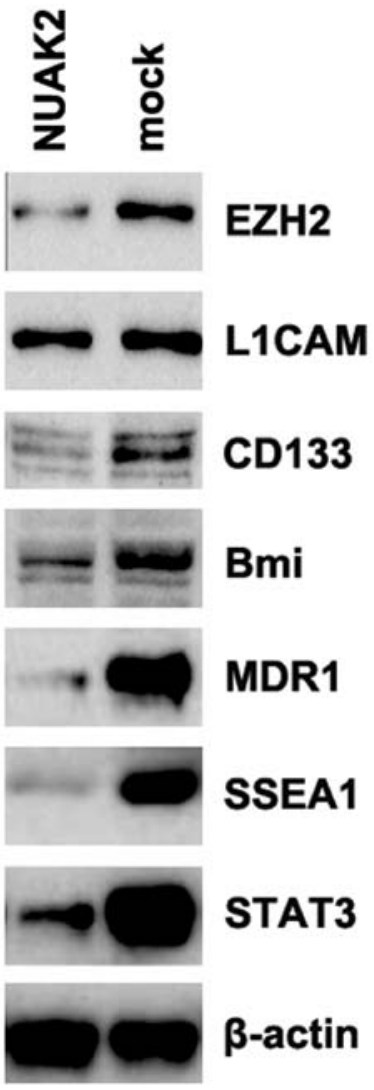

Figure 4. NUAK family, SNF1-like kinase, 2 (NUAK2) regulates cancer stem cell (CSC)-related protein expression in A172 glioblastoma cells. Western blot analysis of EZH2, L1CAM, CD133, Bmi, MDR1, SSEA1 and STAT3 in A172 cells. A172 cells were transfected with a NUAK2 expression plasmid or the empty vector (mock). $\beta$-actin was used as a loading control. $n=3$ experiments.

we performed western blot analysis to measure the expression levels of CSC-related proteins (EZH2, L1CAM, CD133, Bmi, MDR1, SSEA1 and STAT3) (20-26). Our results revealed that NUAK2 promoted the expression of EZH2, CD133, Bmi, MDR1, SSEA1 and STAT3 (Fig. 4). The results revealed that NUAK 2 overexpression was associated with the traits of CSCs in the A172 cells.

miR-143 degrades NUAK2 in A172 glioblastoma cells. Having demonstrated that NUAK2 expression was specifically upregulated and that it promoted the proliferation, migration and invasion of the A172 cells, we then examined the mechanisms promoting NUAK2 expression. miRNAs are a new class of small ( 22 nucleotide) non-coding RNAs that negatively regulate protein-coding gene expression by targeting mRNAs for degradation or translational inhibition (27-29). It has been previously reported that oncogenes are upregulated in cancer, due to a lack of specific miRNAs $(30,31)$.

We hypothesized that NUAK2 was upregulated in glioma due to defects in specific miRNAs in glioblastoma. To further confirm this hypothesis, we used 3 commonly used prediction algorithms, miRanda (http://www.microrna.org/), TargetScan (http://www.targetscan.org) and PicTar (http:// pictar.mdc-berlin.de/) to analyze the 3'UTR of NUAK2. All 3 algorithms predicted that miR-143, miR-23a and miR-23b targeted the 3'UTR of NUAK2 (Fig. 5A). 


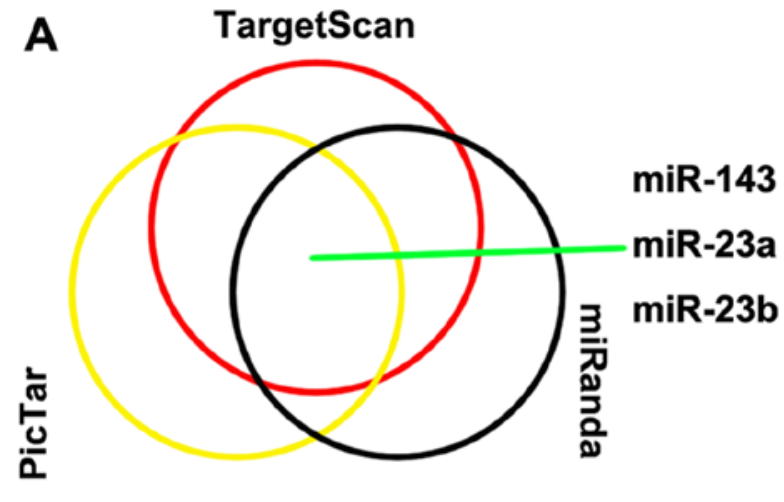

C

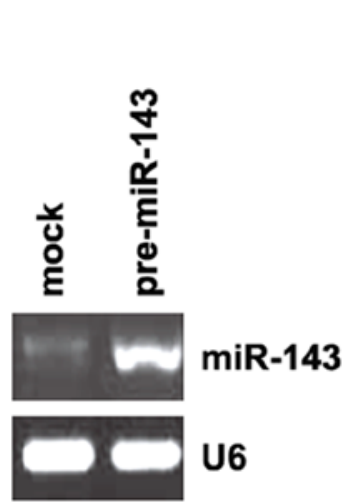

E

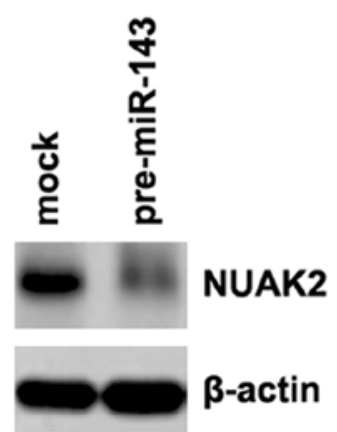

$\mathbf{F}$
B

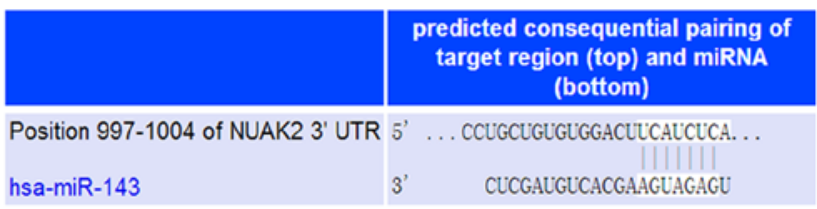

DAPI
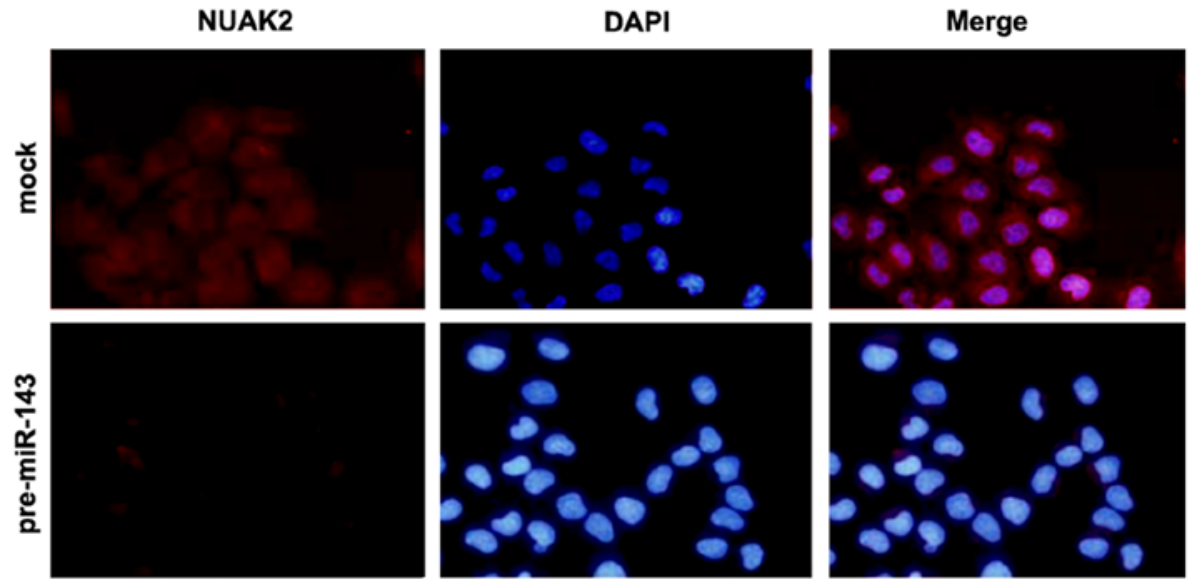

G

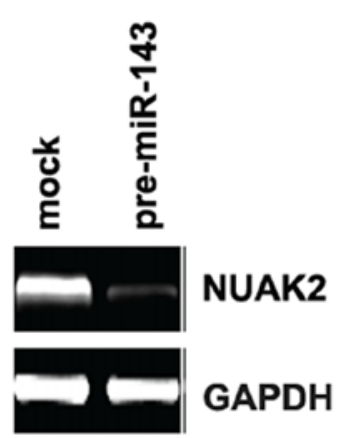

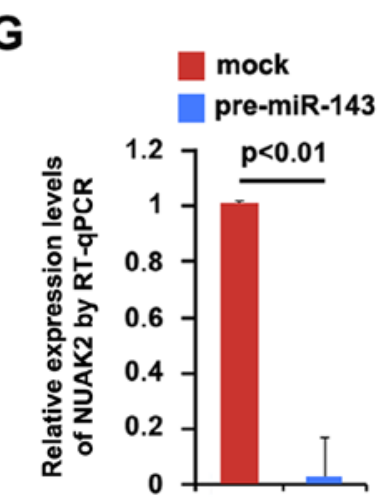

Figure 5. miR-143 degrades NUAK family, SNF1-like kinase, 2 (NUAK2) in glioblastoma A172 cells. (A) Venn diagram showing the predicted miRNA targeting the 3'UTR of NUAK2 mRNA from the databases, TargetSan, miRanda and PicTar. (B) Schematic of predicted miR-143-binding sites in the 3'UTR of NUAK2 mRNA by TargetScan. (C) RT-qPCR of miR-143 in A172 cells transfected with pre-miR-143 or control miR (mock). U6 was used as a loading control. n=3 experiments. (D) Immunofluorescence staining of A172 cells transfected with pre-miR-143 and control miR (mock). n=3 experiments. (E) Western blot analysis of NUAK2 in A172 cells. A172 cells were transfected with pre-miR-143 or control miR (mock). $\beta$-actin was a loading control. $n=3$ experiments. (F) RT-qPCR of NUAK2 in A172 cells. A172 cells were transfected with pre-miR-143 or control miR (mock). GAPDH was a loading control. $n=3$ experiments. (G) RT-qPCR of NUAK2 in A172 cells. A172 cells were transfected with pre-miR-143 or control miR (mock). GAPDH was a loading control. $n=3$ experiments.

Recently, Wang et al reported that miR-143 functions as a tumor suppressor by targeting N-RAS and enhances TMZ-induced apoptosis in glioma (15). Thus, we hypothesized that the upregulation of NUAK2 in glioblastoma is the result of a defect in miR-143. The predicted target sites of miR-143 are illustrated in Fig. 5B.

To determine whether NUAK2 can be downregulated by miR-143, we transfected the A172 cells with pre-miR-143 and RT-qPCR was then performed to detect miR-143 expression in the cells. The results revealed that transfection with pre-miR-143 significantly increased miR-143 expression (Fig. 5C). To determine whether NUAK2 protein expression was affected by miR-143, we performed immunofluorescence staining. The results revealed that NUAK2 protein expression was significantly downregulated following transfection of the A172 cells with pre-miR-143 compared to the cells transfected with control miR (mock; Fig. 5D). Moreover, western blot analysis was performed to measure the protein expression levels of NUAK2 in the A172 cells transfected with pre-miR-143. Consistent with the results of immunofluorescence staining, 
A

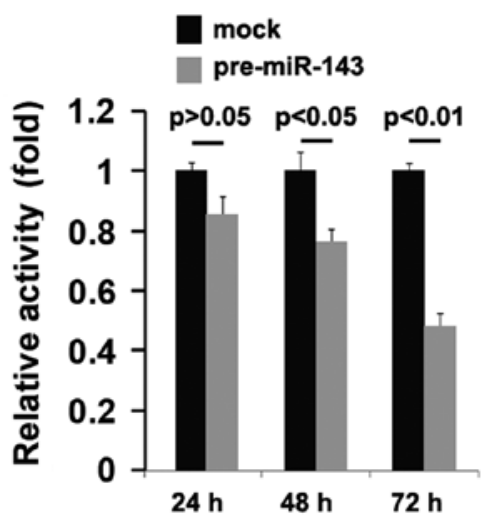

B

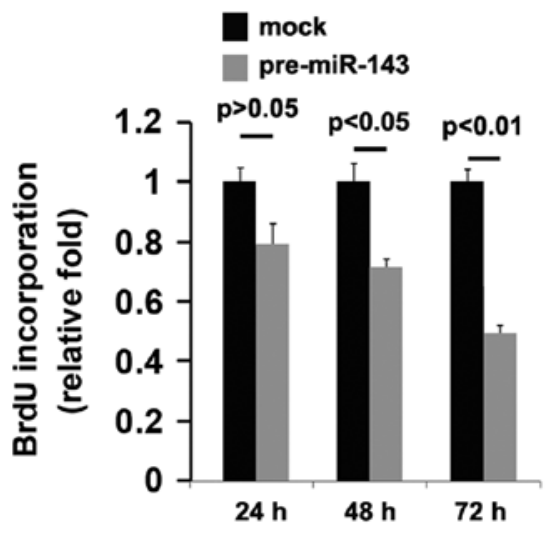

C

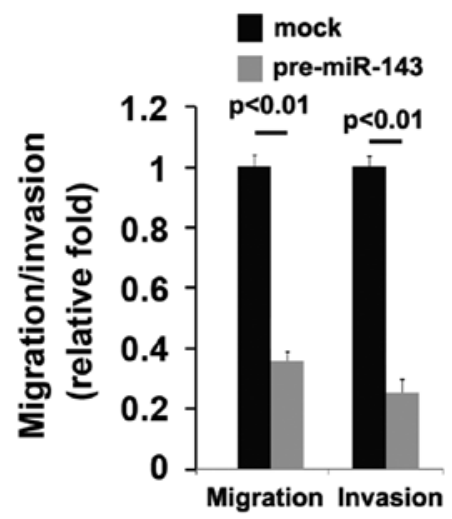

Figure 6. miR-143 functions as a tumor suppressor gene in A172 glioblastoma cells. (A) MTT assay of A172 cells. A172 cells were transfected with premiR-143 or control miR (mock) and cell viability was then measured at the indicated time points by MTT assay. $n=3$ experiments. (B) BrdU incorporation assay for A172 cells. A172 cells were transfected with pre-miR-143 or control miR (mock). $n=3$ experiments. (C) Matrigel invasion assay and Transwell migration assay for A172 cells transfected with pre-miR-143 or control miR (mock). $\mathrm{n}=3$ experiments

we found that NUAK2 protein expression was significantly downregulated following transfection of the A172 cells with pre-miR-143 (Fig. 5E).

As miRNAs can suppress mRNA translation without degrading the mRNA, we also performed RT-qPCR to determine whether miR-143 affects NUAK2 mRNA expression. We transfected the A172 cells with pre-miR-143 and we found that pre-miR-143 evidently degraded NUAK2 mRNA in the A172 cells (Fig. 5F and G). All the results confirmed that
miR-143 degrades NUAK2 and suggest that the overexpression of NUAK 2 is associated with a low expression of miR-143 in gliomas.

miR-143 functions as a tumor suppressorsuppressive gene in A172 glioblastoma cells. We demonstrated that NUAK2 was upregulated in the glioblastoma tissues, and that it promoted the proliferation, migration and invasion of the A172 glioblastoma cells, and that miR-143 degraded NUAK2 in the A172 cells. Moreover, it has been previously demonstrated that miR-143 functions as a tumor suppressor by targeting N-RAS and enhances TMZ-induced apoptosis in glioma (15). Thus, we hypothesized that contrary to NUAK2, miR-203 may inhibit the proliferation, migration and invasion of A172 cells. We demonstrated that miR-143 expression was significantly increased following transfection with pre-miR-143 (Fig. 5C).

Subsequently, we observed the effects of miR-143 on the proliferation, migration and invasion of the A172 cells. An MTT assay, a migration assay and an invasion assay were performed on the A172 cells. We demonstrated that miR-143 significantly suppressed the proliferation rate of the A172 cells and that the decrease in cell proliferation was time-dependent (Fig. 6A). This was further confirmed by BrdU incorporation assay, which indicated that transfection with pre-miR-143 resulted in decreased DNA synthesis activity per viable cell in the A172 cells, also in a time-dependent manner (Fig. 6B).

Given that miR-143 inhibited A172 cell proliferation, we then sought to determine whether miR-143 would have an impact on the migration and invasion of A172 cells. The migration and invasion assay of A172 cells revealed that miR-143 not only suppressed the migration, but also and inhibited the invasion of the A172 cells (Fig. 6C).

\section{Discussion}

Gains or amplifications of the long arm of chromosome 1 are among the frequent chromosomal abnormalities in various types of cancer, and gains in the region spanning 1q31-1q32 are the most frequent abnormalities in these loci. The observation that the gain of the 1q32 locus is shared by various types of cancer emphasizes the importance of this locus for cancer development and tumor progression in general (32). NUAK2 resides at 1q32 and public databases, such as GeneCards (www.genecards.org) indicate that NUAK2 is highly expressed in various types of cancer, including cancers of the lymphoid tissues, lungs and breasts, implying that it may be associated with cancer development and tumor progression $(6,11,12)$. Recently, Namiki et al (33) reported that the AMPK-related kinase, NUAK2, affects tumor growth, migration and the clinical outcome of human melanoma cells, which further confirms the role of NUAK 2 in cancer. However, to the best of our knowledge, there is no study available to date on the role of NUAK2 in glioma.

In this study, we demonstrated that NUAK 2 expression was upregulated in glioma tissues compared with matched adjacent normal tissues and that its expression was increased in the advanced stages of the disease, suggesting that it is associated with the development and progression of glioma. NUAK 2 overexpression promoted the proliferation, migration and invasion of the glioma cells, while knockdown in vitro experiments using plasmids containing shRNA targeting 
NUAK2 revealed that NUAK2 has a significant impact on the proliferation and migration of glioblastoma cells.

A subset of cancer cells within some tumors, CSCs, may drive the growth, multiple drug resistance and metastasis of these tumors (34-36). Understanding the pathways that regulate the proliferation, self-renewal, survival and differentiation of malignant stem cells may shed light on the mechanisms that lead to cancer development and may provide better modes of treatment.

We found that NUAK2 downregulated the expression of CSC-associated genes. It has been demonstrated that EZH2 is essential for glioblastoma CSC maintenance (20); the stem cell marker, CD133, has been found to affect clinical outcomes in glioma patients (21); Bmi-1 has also been shown to promote stem cell self-renewal (22); it has been demonstrated that the gene expression of MDR1 in glioblastoma stem cells is increased (23); SSEA-1 has been demonstrated to be an enrichment marker for tumor-initiating cells in human glioblastoma (24); evidence suggests that STAT3 is required for the maintenance of multipotency in glioblastoma stem cells (25). We demonstrated that NUAK2 upregulated EZH2, CD133, Bmi-1, MDR1, SSEA-1 and STAT3 protein expression, implying that NUAK2 overexpression may promote the production of CSCs.

Consistent with the results of a previous study demonstrating that miR-143 functions as a tumor suppressor by targeting N-RAS (15), in this study, we demonstrated that miR-143 degraded NUAK 2 mRNA and contrary to the role of NUAK2, it inhibited the proliferation, migration and invasion of glioblastoma cells. Thus, from our data, it can be concluded that miR-143 inhibits oncogenic traits by degrading NUAK2 in glioblastoma cells.

\section{References}

1. Deorah S, Lynch CF, Sibenaller ZA and Ryken TC: Trends in brain cancer incidence and survival in the United States: surveillance, epidemiology, and end results program, 1973 to 2001. Neurosurg Focus 20: E1, 2006.

2. Stupp R, Mason WP, van den Bent MJ, Weller M, Fisher B, Taphoorn MJ, Belanger K, Brandes AA, Marosi C, Bogdahn U, et al; European Organisation for Research and Treatment of Cancer Brain Tumor and Radiotherapy Groups; National Cancer Institute of Canada Clinical Trials Group: Radiotherapy plus concomitant and adjuvant temozolomide for glioblastoma. N Engl J Med 352: 987-996, 2005.

3. Stupp R, Hegi ME, Mason WP, van den Bent MJ, Taphoorn MJ, Janzer RC, Ludwin SK, Allgeier A, Fisher B, Belanger K, et al; European Organisation for Research and Treatment of Cancer Brain Tumour and Radiation Oncology Groups; National Cancer Institute of Canada Clinical Trials Group: Effects of radiotherapy with concomitant and adjuvant temozolomide versus radiotherapy alone on survival in glioblastoma in a randomised phase III study: 5-year analysis of the EORTC-NCIC trial. Lancet Oncol 10: 459-466, 2009.

4. Hardie DG: AMP-activated/SNF1 protein kinases: conserved guardians of cellular energy. Nat Rev Mol Cell Biol 8: 774-785, 2007.

5. Kato K1, Ogura T, Kishimoto A, Minegishi Y, Nakajima N, Miyazaki M and Esumi H: Critical roles of AMP-activated protein kinase in constitutive tolerance of cancer cells to nutrient deprivation and tumor formation. Oncogene 21: 6082-6090, 2002.

6. Lizcano JM, Göransson O, Toth R, Deak M, Morrice NA, Boudeau J, Hawley SA, Udd L, Mäkelä TP, Hardie DG and Alessi DR: LKB1 is a master kinase that activates 13 kinases of the AMPK subfamily, including MARK/PAR-1. EMBO J 23: $833-843,2004$
7. Manning G, Whyte DB, Martinez R, Hunter T and Sudarsanam S: The protein kinase complement of the human genome. Science 298: 1912-1934, 2002.

8. Martin MJ, Carling D and Marais R: Taking the stress out of melanoma. Cancer Cell 15: 163-164, 2009.

9. Ashrafian H: Cancer's sweet tooth: the Janus effect of glucose metabolism in tumorigenesis. Lancet 367: 618-621, 2006.

10. Legembre P, Schickel R, Barnhart BC and Peter ME: Identification of SNF1/AMP kinase-related kinase as an NF-kappaB-regulated anti-apoptotic kinase involved in CD95-induced motility and invasiveness. J Biol Chem 279: 46742-46747, 2004.

11. Lefebvre DL, Bai Y, Shahmolky N, Sharma M, Poon R, Drucker DJ and Rosen CF: Identification and characterization of a novel sucrose-non-fermenting protein kinase/AMP-activated protein kinase-related protein kinase, SNARK. Biochem J 355: 297-305, 2001.

12. Zagórska A, Deak M, Campbell DG, Banerjee S, Hirano M, Aizawa S, Prescott AR and Alessi DR: New roles for the LKB1-NUAK pathway in controlling myosin phosphatase complexes and cell adhesion. Sci Signal 3: ra25, 2010.

13. Bartel DP: MicroRNAs: genomics, biogenesis, mechanism, and function. Cell 116: 281-297, 2004.

14. Baek D, Villén J, Shin C, Camargo FD, Gygi SP and Bartel DP: The impact of microRNAs on protein output. Nature 455: 64-71, 2008.

15. Wang L, ShiZM, Jiang CF, Liu X, Chen QD, Qian X, Li DM, Ge X, Wang XF, Liu LZ, et al: MiR-143 acts as a tumor suppressor by targeting N-RAS and enhances temozolomide-induced apoptosis in glioma. Oncotarget 5: 5416-5427, 2014.

16. Heck S, Rom J, Thewes V, Becker N, Blume B, Sinn HP, Deuschle U, Sohn C, Schneeweiss A and Lichter P: Estrogenrelated receptor alpha expression and function is associated with the transcriptional coregulator AIB1 in breast carcinoma. Cancer Res 69: 5186-5193, 2009.

17. Bao B, Wang Z, Ali S, Ahmad A, Azmi AS, Sarkar SH, Banerjee S, Kong D, Li Y, Thakur S and Sarkar FH: Metformin inhibits cell proliferation, migration and invasion by attenuating CSC function mediated by deregulating miRNAs in pancreatic cancer cells. Cancer Prev Res (Phila) 5: 355-364, 2012.

18. Yu F, Li J, Chen H, Fu J, Ray S, Huang S, Zheng H and Ai W: Kruppel-like factor 4 (KLF4) is required for maintenance of breast cancer stem cells and for cell migration and invasion. Oncogene 30: 2161-2172, 2011.

19. Tang SN, Singh C, NallD, Meeker D, Shankar S and Srivastava RK: The dietary bioflavonoid quercetin synergizes with epigallocathechin gallate (EGCG) to inhibit prostate cancer stem cell characteristics, invasion, migration and epithelial-mesenchymal transition. J Mol Signal 5: 14, 2010

20. Suvà ML, Riggi N, Janiszewska $M$, Radovanovic I, Provero $\mathrm{P}$, Stehle JC, Baumer K, Le Bitoux MA, Marino D, Cironi L, et al: $\mathrm{EZH} 2$ is essential for glioblastoma cancer stem cell maintenance. Cancer Res 69: 9211-9218, 2009.

21. Zeppernick F, Ahmadi R, Campos B, Dictus C, Helmke BM, Becker N, Lichter P, Unterberg A, Radlwimmer B and Herold-Mende CC: Stem cell marker CD133 affects clinical outcome in glioma patients. Clin Cancer Res 14: 123-129, 2008.

22. Godlewski J, Nowicki MO, Bronisz A, Williams S, Otsuki A, Nuovo G, Raychaudhury A, Newton HB, Chiocca EA and Lawler S: Targeting of the Bmi-1 oncogene/stem cell renewal factor by microRNA-128 inhibits glioma proliferation and selfrenewal. Cancer Res 68: 9125-9130, 2008.

23. Nakai E, Park K, Yawata T, Chihara T, Kumazawa A, Nakabayashi H and Shimizu K: Enhanced MDR1 expression and chemoresistance of cancer stem cells derived from glioblastoma. Cancer Invest 27: 901-908, 2009.

24. Son MJ, Woolard K, Nam DH, Lee J and Fine HA: SSEA-1 is an enrichment marker for tumor-initiating cells in human glioblastoma. Cell Stem Cell 4: 440-452, 2009.

25. Sherry MM, Reeves A, Wu JK and Cochran BH: STAT3 is required for proliferation and maintenance of multipotency in glioblastoma stem cells. Stem Cells 27: 2383-2392, 2009.

26. Bao S, Wu Q, Li Z, Sathornsumetee S, Wang H, McLendon RE, Hjelmeland $\mathrm{AB}$ and Rich JN: Targeting cancer stem cells through L1CAM suppresses glioma growth. Cancer Res 68: 6043-6048, 2008.

27. Lee RC, Feinbaum RL and Ambros V: The C. elegans heterochronic gene lin-4 encodes small RNAs with antisense complementarity to lin-14. Cell 75: 843-854, 1993.

28. Pasquinelli AE, Reinhart BJ, Slack F, Martindale MQ, Kuroda MI, Maller B, Hayward DC, Ball EE, Degnan B, Müller P, et al: Conservation of the sequence and temporal expression of let-7 heterochronic regulatory RNA. Nature 408: 86-89, 2000. 
29. Reinhart BJ, Slack FJ, Basson M, Pasquinelli AE, Bettinger JC, Rougvie AE, Horvitz HR and Ruvkun G: The 21-nucleotide let-7 RNA regulates developmental timing in Caenorhabditis elegans. Nature 403: 901-906, 2000

30. Saito Y, Liang G, Egger G, Friedman JM, Chuang JC, Coetzee GA and Jones PA: Specific activation of microRNA-127 with downregulation of the proto-oncogene BCL6 by chromatin-modifying drugs in human cancer cells. Cancer Cell 9: 435-443, 2006.

31. Varambally S, Cao Q, Mani RS, Shankar S, Wang X, Ateeq B, Laxman B, Cao X, Jing X, Ramnarayanan $\mathrm{K}$, et al: Genomic loss of microRNA-101 leads to overexpression of histone methyltransferase EZH2 in cancer. Science 322: 1695-1699, 2008.

32. Corson TW, Huang A, Tsao MS and Gallie BL: KIF14 is a candidate oncogene in the 1q minimal region of genomic gain in multiple cancers. Oncogene 24: 4741-4753, 2005.
33. Namiki T1, Tanemura A, Valencia JC, Coelho SG, Passeron T, Kawaguchi M, Vieira WD, Ishikawa M, Nishijima W, Izumo T, et al: AMP kinase-related kinase NUAK2 affects tumor growth, migration, and clinical outcome of human melanoma. Proc Natl Acad Sci USA 108: 6597-6602, 2011.

34. Bjerkvig R, Tysnes BB, Aboody KS, Najbauer J and Terzis AJ: Opinion: the origin of the cancer stem cell: current controversies and new insights. Nat Rev Cancer 5: 899-904, 2005.

35. Reya T, Morrison SJ, Clarke MF and Weissman IL: Stem cells, cancer, and cancer stem cells. Nature 414: 105-111, 2001.

36. Donnenberg VS and Donnenberg AD: Multiple drug resistance in cancer revisited: the cancer stem cell hypothesis. J Clin Pharmacol 45: 872-877, 2005. 